\title{
Numerical Investigation of Two-Phase Air/Oil Flow in High-speed Angular-contact Ball Bearing Chamber
}

\author{
Hong-bin Liu, ${ }^{1, \text { a) }}$, Gong-ping Liu ${ }^{1, \text { b) }}$, Yun-bin $\mathrm{Li}^{1, \text { c) }}$ and L.Wang ${ }^{2)}$ \\ ${ }^{1}$ School of Mechatronics Engineering, Henan University of Science and Technology, Luoyang 471003, \\ China. \\ ${ }^{2}$ Southampton University, Southampton, UK \\ a)hongbin-liu@163.com \\ b)gongpingliu@126.com \\ c)lyb199403@163.com
}

Keywords: bearing chamber; air/oil two-phase flow; VOF model; DN value

\begin{abstract}
Based on the model of gas liquid two-phase flow of lubrication oil in bearing chamber, the paper calculates the flow characteristics of the lubricating oil in the simplified model of the high-speed angular contact ball bearing chamber by VOF method and MRF model, obtains the pressure and distribution of lubricant oil. The cause of the influence of the lubricating oil into the chamber and the influence of the rotational speed of the bearing and lubricating oil inlet flow parameters such as the impact on the oil volume fraction are analyzed. The results show that bearing high-speed rotating stage, the two phase flow field in the bearing chamber between the bearing rings has the characteristics of periodic pressure. The volume fraction of the oil in the chamber decreases with the increase of the bearing speed, and is increased with speeding up the lubrication flow rate nonlinearly, which provides a theoretical basis for the design of high DN bearing lubrication.
\end{abstract}

\section{Introduction}

Angular contact ball bearings can withstand radial and axial load at the same time, and have excellent speed performance, widely used in aviation engine spindle, high-speed spinning spindle, and other mechanical parts. Its stable working condition is very important to the whole system. Due to the structure of oil injection lubrication is easier to achieve, and has good lubrication effect [1]. Therefore, it is widely used in high speed angular contact ball bearings. Because of bearing high speed rotating, lubricating oil into the bearing chamber by the rolling element, cage stirring and centrifugal force, the formation of the flow state of gas-liquid coexistence with the chamber air, namely the gas liquid two phase flow. Gas-liquid two phase flow in the flow and distribution of bearing cavity for bearing lubrication performance and service life has an important influence.

In recent years, domestic and foreign scholars in the field of bearing chamber two-phase flow launched a series of research work. Glahn and Wittig[2] were used to study the effects of interfacial shear stress, gravity and heat transfer on the oil film motion in the bearing chamber. Coe, etc.[3] found that the bearing oil chamber volume fraction from $2 \%$ to $3 \%$, the bearing temperature rise calculation results are comparatively consistent with the experiment. Hadden[4], such as through a lot of experimental research is recommended to use the oil volume fraction below $5 \%$ for the bearing lubrication and cooling effect is better. Chen, etc.[5] studied the aircraft engine bearing chamber gas liquid two phase flow field in the deformation and movement of the droplet. The numerical simulation of gas liquid two phase flow in the oil lubricated rolling bearing was carried out by $\mathrm{Hu}$, et al[6], which provided the basis for optimizing the jet lubrication device and enhancing the cooling effect. These results are of great significance for understanding the gas-liquid two-phase flow in the bearing chamber. However, for high speed rolling bearing ring oil lubrication between 
research rarely seen reports that the present study focused on the determination of lubrication parameters, the formation and distribution of oil film, production and the movement of the droplet. There is a lack of understanding the gas-liquid two-phase flow in the internal bearings.

Based on the angular contact ball bearing 7306 as the research object, the VOF method was used to track gas-liquid interface and description bearing parts movement of MRF multiple coordinate system. Analyze the pressure distribution in the bearing chamber, the oil volume fraction with bearing rotational speed and the inlet flow rate of lubricating oil. To improve rolling bearings under high DN value provides the theory basis for oil lubrication.

\section{Mathematical Model and Control Equation}

\section{VOF Method and MRF Model}

While the high-speed angular contact ball bearing in the gas-liquid two phase flow state, but the chamber flow approximation think there are two incompatible and incompressible fluid flow system, consisting of each phase is still meet the basic control equation of fluid. This paper uses the VOF method to introduce a variable $\varphi$, namely the calculation of the volume fraction of unit (the air for the first phase, the second phase of lubricating oil). If $\varphi_{l}=0$, the unit all the air; if $\varphi_{l}=1$, the unit all occupied by oil liquid; if $0<\varphi_{l}<1$, the unit is at the gas-liquid interface, unit for gas-liquid two-phase fluid; in each control volume, the volume fraction of two phase and 1.

High-speed angular contact ball bearing in high speed, bearing inner ring, cage and rolling element with their respective speed of revolution, the position of the nozzle is fixed, in order to accurately simulate the bearing chamber form of exercise, two sets of coordinate system is adopted to improve the movement description, namely the method of multiple reference frame (MRF)[7]. When the rotation basin is rotated with angular velocity $\omega$, the relationship between the relative velocity $\boldsymbol{v}_{r}$ of the point of the vector at $\boldsymbol{r}$ and the absolute velocity $\boldsymbol{v}$ in the fixed coordinate system can be expressed as:

$$
v=v_{r}+\omega \times r
$$

Rotating coordinates of fluid mass, momentum and energy conservation equation can be described as[8]:

$$
\begin{gathered}
\frac{\partial \boldsymbol{\rho}}{\partial t}+\nabla \boldsymbol{\bullet} \boldsymbol{\rho} \boldsymbol{v}_{r}=0 \\
\frac{\partial}{\partial t}\left(\boldsymbol{\rho} \boldsymbol{v}_{r}\right)+\nabla \bullet\left(\boldsymbol{\rho} \boldsymbol{v}_{r} \boldsymbol{v}_{r}\right)+\boldsymbol{\rho}\left(2 \boldsymbol{\omega} \times \boldsymbol{v}_{r}+\boldsymbol{\omega} \times \boldsymbol{\omega} \times \boldsymbol{r}\right)= \\
-\nabla P+\nabla \cdot\left[\boldsymbol{\mu}\left(\nabla \boldsymbol{v}+\nabla \boldsymbol{v}^{\mathrm{T}}\right)\right]+\boldsymbol{F}
\end{gathered}
$$

In the formula, $\boldsymbol{\omega} \times \boldsymbol{v}_{\boldsymbol{r}}$ and $\boldsymbol{\omega} \times \boldsymbol{\omega} \times \boldsymbol{r}$ respectively represent the Coriolis acceleration and the centripetal acceleration which are relative to the rotational motion.

\section{Mesh Generation and Boundary Conditions}

Model No. 7306 angular contact bearing was chosen as the research object. The bearing parameters: outer diameter $72 \mathrm{~mm}$, inner diameter $30 \mathrm{~mm}$, width $19 \mathrm{~mm}$, contact angle $15 \mathrm{deg}$ and 12 rolling bodies. In order to calculate more accurate computational resource in limited circumstances, need to simplify the model, the inner and outer rings to retain only the inner wall of the rolling element and the cage of the shell. Considering the motion characteristics of the rolling bearing was analyzed with periodic conditions, the simplified model is sketched as Figure 1. Entity model import ICEM, although the simplified model, the internal structure of bearing irregular partition structure grid is difficult, so the use of unstructured tetrahedral meshes were divided, the bearing inner and outer ring raceway surface partial encryption. The overall model mesh number was 2867328, and the mesh model was shown in figure 2 . The nozzle entrance by mass flow boundary conditions, bearing small end face and the large end face pressure inlet and outlet boundary conditions respectively, temperature is set to the default; the inner ring and the wall of the rotating part were set as the rotating boundaries; the revolution speed of the rolling element and cage is denoted by the following[9]: 


$$
n_{m}=\frac{n_{i}}{2}\left(1-\frac{D_{w} \cos \alpha}{d_{m}}\right)
$$

Where, $n_{i}$ is the speed of the inner ring; $d_{m}$ is the diameter of the rolling element; $\alpha$ is the contact angle; and $D_{w}$ is the pitch diameter of the bearing. The standard wall function was applied to the area near the wall, while the other walls were treated as adiabatic, solid and non-slipping boundaries. The rotational speed was decided as per the designed working conditions of the high-speed rolling shaft. On software platform FLUENT, the second-order windward format of discrete momentum equation and turbulence equation were deployed for calculation. The pressure term was converted into "PRESTO!" discrete format. Finally the SIMPLE algorithm was employed to find the solutions for the discrete algebraic equation. The convergence criteria were defined as follows: when the residual value fell below $10^{-4}$, the net flux from inlet to outlet should reach about $0.1 \%$.

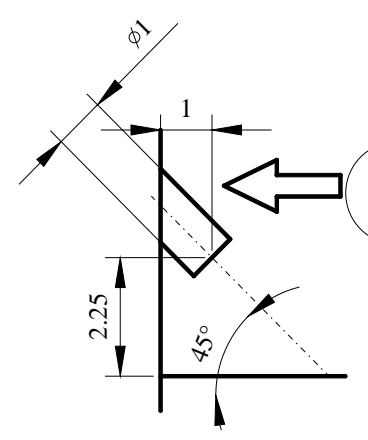

FIGURE1. Nozzle position
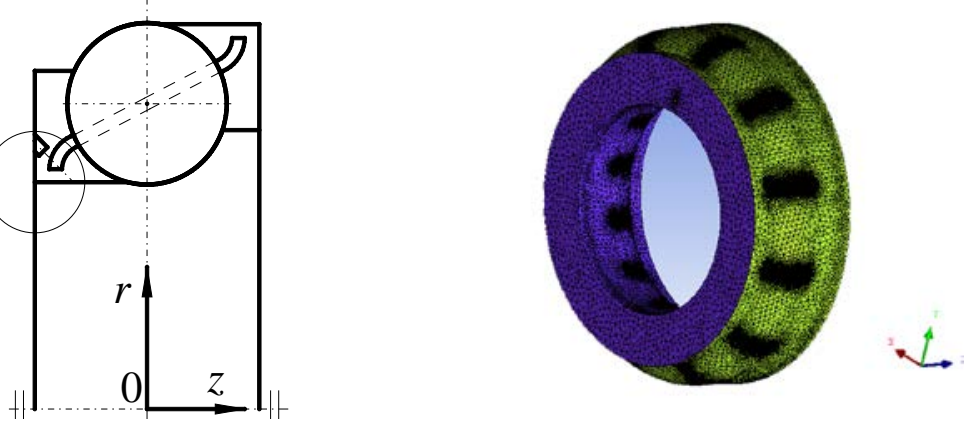

FIGURE 2. Whole mesh model of the gas-liquid two-phase Flow

\section{Results and Analysis}

\section{Pressure Distribution between Bearing Rings}

The pressure of two phase flow field in bearing chamber is an important parameter to analyze the penetration mechanism of bearing oil. Figure3 shows bearing rotating speed at $1.0 \times 10^{4} \mathrm{r} / \mathrm{min}$, $5.0 \times 10^{4} \mathrm{r} / \mathrm{min}, 1.0 \times 10^{5} \mathrm{r} / \mathrm{min}$, the relative circumferential pressure distribution of bearing ring when bearing rotates for one week. The azimuth angle of the nozzle $\alpha$ was set zero, the location is shown in figure 1. Take lubricating oil flow direction as positive direction, take a cross section every 10 degrees along the circumference, and calculate the pressure value of the weighted average.

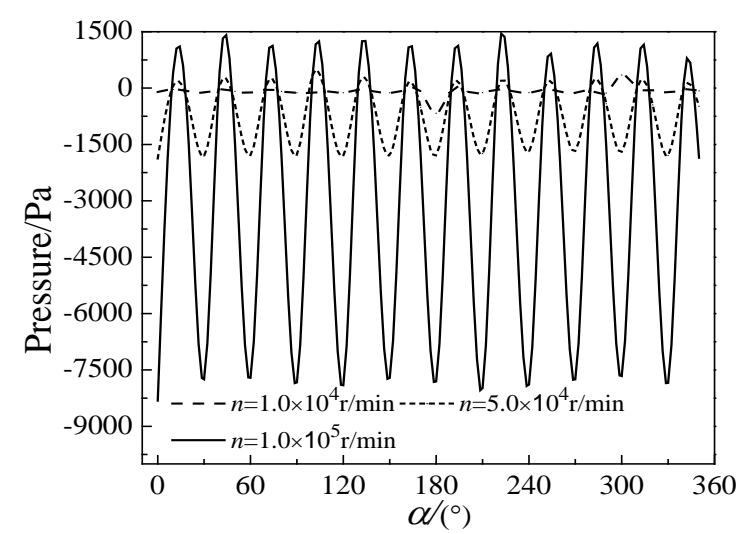

FIGURE3. Circumferential relative pressure between rings

It can be seen from figure 3 that annular pressure between the two phases of the bearing rings had characteristics of periodicity, which was mainly because of the small amount of lubricating oil into chamber, and pressure between the rings was still dominated by the pressure of gas. When speed at $1.0 \times 10^{4} \mathrm{r} / \mathrm{min}$, you can clearly see the pressure between the bearing rings was basically in 
one bar pressure, then the pressure of the lubricating oil into little effect; with the increase of speed, the location of various pressure fluctuations. At the same time, the peak value of pressure increases gradually with increase of the speed, especially for the increase of the positive pressure, it will be more detrimental to the lubrication oil into the lubrication area. Therefore, for low speed, the circumferential pressure change of the bearing ring was very small and can be neglected; at higher speed, it exhibits periodic fluctuation, and influence on lubrication oil entry cannot be ignored. The energy loss and influence of the gas flow in lubricating oil cannot reach lubrication area through the positive pressure region. The circumferential pressure between high speed rolling bearing rings has a great influence on oil injection quantity.

\section{Volume Distribution of Oil in Bearing Chamber}

Figure 4 were the nephograms for oil volume fraction distribution at different time instants when rotating speed at $1.0 \times 10^{5} \mathrm{r} / \mathrm{min}$. In the early stages, the motion curve of the lubricating oil on the bearing wall was like a thread, the volume fraction of oil in the vicinity of the nozzle was larger, as shown in Figure 4(a). With the increase of the oil injection time, the amount of oil in the bearing chamber also increased. Some of the oil in the bearing chamber presented like droplets, and some merged into a band that formed the oil film, as shown in Figure 4(b). By the time of 0.000945s, the bearing had experienced one revolution. As shown in Figure 4(c), oil entering the bearing ring can still partially merge into bands and lubricate the bearing in the form of oil films.

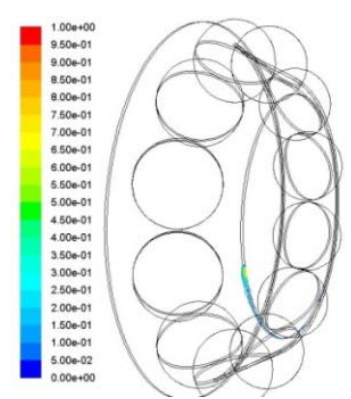

(a) Oil volume cloud image at $0.00009 \mathrm{~s}$

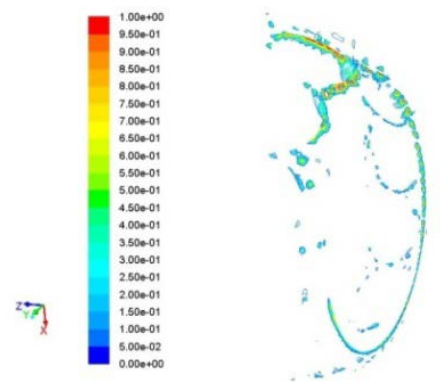

(b) Oil volume cloud image at $0.000718 \mathrm{~s}$

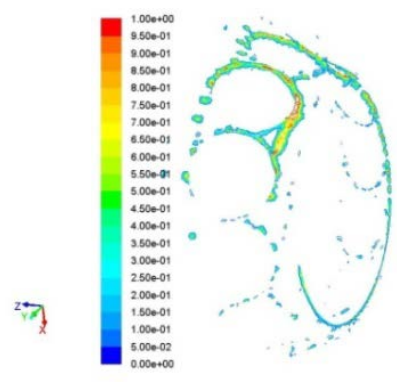

(c) Oil volume cloud image at $0.000945 \mathrm{~s}$

FIGURE4. Oil distribution when rotating speed at $1.0 \times 10^{5} \mathrm{r} / \mathrm{min}$

\section{Effect of Bearing Speed and Oil Supply on Oil Volume Fraction in Bearing Chamber}

Bearing speed and oil supply are important factors affecting volume fraction of oil in bearing chamber. Figure 5 was steady under different rotational speed when oil injection quantity $q_{v}(\mathrm{~L} / \mathrm{min})$ for influence of oil volume fraction $\varphi$ of bearing rings. With the increase of injection quantity of $q_{v}$, oil volume fraction $\varphi$ increases nonlinearly. When bearing speed $n=1.0 \times 10^{4} \mathrm{r} / \mathrm{min}$ with oil injection quantity is $q_{v}=7 \mathrm{~L} / \mathrm{min}$ bearing ring between oil volume fraction reached $2 \%$, basic $3.0 \times 10^{4} \mathrm{r} / \mathrm{min}$ following with the increase of fuel injection quantity of basic oil volume fraction can reach $2 \%$. And rotational speed in more than $3.0 \times 10^{4} \mathrm{r} / \mathrm{min}$ and then improve the fuel injection quantity bearing ring between the oil volume fraction increase is not obvious. Accordingly, when low speed by increasing injection can improve bearing chamber oil volume fraction, but as speed increases, by increasing volume fraction of oil injection quantity the bearing chamber does not improve significantly, it need to be improved by other means. Here mainly consider injection parameters of oil injection quantity on influence of oil volume fraction of bearing chamber. 


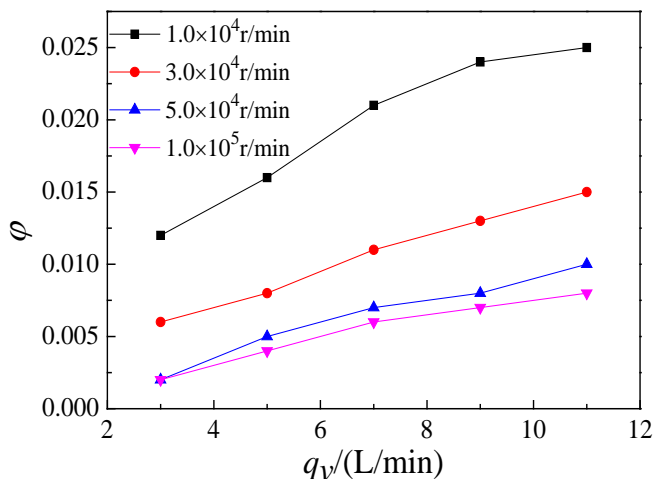

FIGURE5. Supply oil affect the oil volume fraction

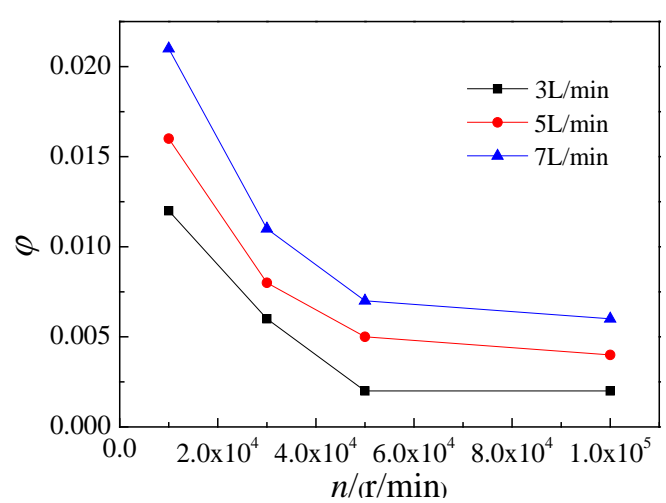

FIGURE6. Rotating speed affect the

oil volume fraction

Figure 6 described the effect of rotation speed on the volume fraction of oil. It is shown that there was a small difference in the volume fraction of the lubricating oil in the high speed stage, and it decreases with the increase of the rotating speed, and finally tends to be stable. The oil volume fraction is too small, mainly is the tangential velocity of airflow makes most gathered in lubricating oil bearing small end face do circumferential movement. And this part of the oil is easily affected by the centrifugal force from the bearing small end face left out, so enter the chamber lubricating oil is too small. In addition, when the oil injection quantity $q_{v}=3 \mathrm{~L} / \mathrm{min}$ and rotating speed at $1.0 \times 10^{4}$ $\mathrm{r} / \mathrm{min}$ the oil volume fraction $\varphi$ is only $1.2 \%$, did not reach the oil volume fraction of ideal, $3 \mathrm{~L} / \mathrm{min}$ indicates that the oil injection quantity is too small. However, with the increase of the initial oil injection quantity, oil volume fraction increases not obviously, the oil injection quantity $q_{v}=7 \mathrm{~L} / \mathrm{min}$ and speed for $1 \times 10^{4} \mathrm{r} / \mathrm{min}$ the oil volume fraction increased to $2.1 \%$. It can be seen that the amount of oil injection in the initial stage of oil injection has certain influence on the oil volume fraction in the bearing chamber.

\section{Test Validation}

The test results show that the bearing speed and the amount of oil supply affect oil volume fraction in the bearing chamber and the results are compared with the simulation results to verify the correctness of the simulation results.

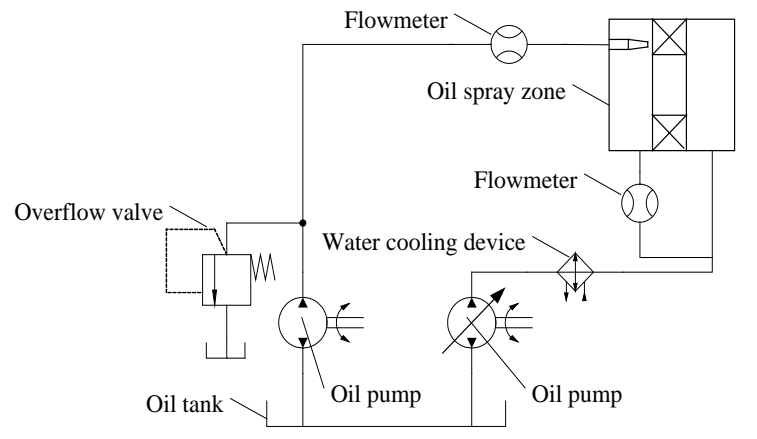

FIGURE7. Schematic diagram of the oil lubrication system

Figure 7 was the schematic diagram for the oil lubrication system. First, the test bearing kept running at low speed for $12 h$ (working conditions: $n=1,000 \mathrm{r} / \mathrm{min}, q_{v}=0.07 \mathrm{~L} / \mathrm{min}$ ). Then $n$ and $q_{v}$ were adjusted to the specified values. Once the bearing ran stably, the flow rates of the oil inlet pipe and of the oil return pipe were recorded every $1 \mathrm{~min}$. In total ten records were acquired for each working condition. Finally, data processing was conducted. The difference between inlet flow rate and return flow rate was the flow rate of the lubricating oil injected into the bearing ring. The percentage of the amount of lubricating oil in the bearing ring over the volume of the bearing chamber was defined as the volume fraction of oil between the bearing rings. 


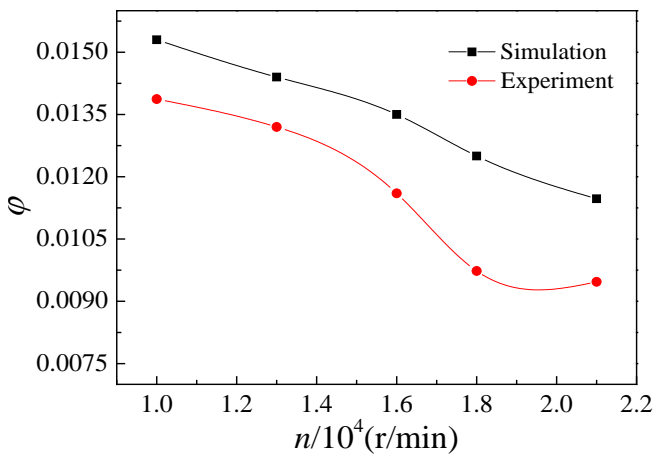

FIGURE8. Oil volume fraction in the bearing chamber with change of rotation speed

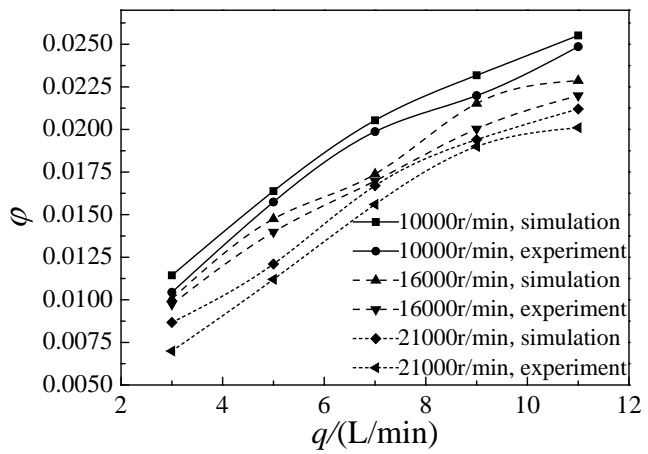

FIGURE9. Oil volume fraction in the bearing chamber with change of oil injection quantity

Figure 8 shows the $q_{v}=3 \mathrm{~L} / \mathrm{min}$ amount of oil in the oil volume fraction with the speed variation, and compared with the results obtained by simulation. Slightly smaller than the simulation data obtained with tests, the reason was that in the process of test machine exists some shortage, lead to system has certain measurement error. Though the tested data showed some deviation from the simulated data, their trends were basically identical. With the relatively small error, the experimental results can be considered to coincide with the simulation results.

Figure9 shows the oil volume fraction under different speed with the injection amount of variation. The test results proved that, the oil volume fraction increased nonlinearly with the increase of $q_{v}$, but decreased for a higher the speed. When $q_{v}$ climbed to a certain value, the oil volume fraction ceased increasing. This trend was basically consistent with the simulation. The tested results were also numerically slightly smaller than the simulated results. The reason for this error was the same as the description above.

\section{Conclusion}

In this paper, the VOF model and the MRF method were used to simulate the gas-liquid twophase flow in the high-speed angular contact ball bearing. The annular pressure between two phases of the bearing ring has the characteristics of periodicity, mainly because the lubricating oil into the bearing chamber quantity is less, and the pressure between the rings is still dominated by the pressure of the gas. Lubricating oil energy loss in the process of movement itself and the influence of transverse gas flow makes it did not reach the lubrication region through the positive pressure area. The volume fraction of the oil in the bearing chamber decreases with the increase of the bearing speed, and increases with the increase of the oil injection quantity, which is nonlinear relationship. The simulation results are consistent with the test results, and correctness of the simulation results is verified indirectly.

\section{Acknowledgments}

The research was sponsored by the Tribology Science Found of State Key Laboratory of Tsinghua University, China(SKLTKF13A03) and the National Natural Science Foundation of China(U1404517).

\section{References}

[1] Pinel S 1, Signer H R, Zaretsky E V. Comparison between oil-mist and oil-jet lubrication of high-speed, small-bore, angular-contact ball bearings[J]. Tribology Transactions, 2001, 44(3): 327-338.

[2] Glahn A, Wittig S, Two-phase air/oil flow in aero-engine bearing chambers-assessment of an analytical prediction method for the internal wall heat transfer[J]. Internal Journal of Rotating Machinery, 1999, 5(3): 155-165. 
[3] Coe H H, Zaretsky E V. Predicted and experimental performance of jet-lubricated 120millimeter-bore ball bearings operating to 2.5 million DN NASA, TP-1196[R]. Washington D. C. : NASA, 1978.

[4] Hadden G B, Kleckner R J, Ragen M A, et a1. Steady state and transient thermal analysis of a shaft bearing system including ball, cylindrical, and tapered roller bearings NASA, CR165365[R]. Washington D. C.: NASA, 1981.

[5] B. Chen, G.D. Chen, H.C. Sun, et al. Effect of oil droplet deformation on its deposited characteristics in an aero-engine bearing chamber, Proc. Inst. Mech.Eng. G 228 (2) (2012) 206218.

[6] $\mathrm{Hu} \mathrm{J}, \mathrm{Wu} \mathrm{W}, \mathrm{Wu} \mathrm{M}$, et al. Numerical investigation of the air-oil two-phase flow inside an oiljet lubricated ball bearing[J]. International Journal of Heat \& Mass Transfer, 2014, 68:85-93.

[7] LUO J Y, ISSA R I, GoSMAN A D. Prediction of impeller induced flows in mixing vessels using multiple frames of reference[J]. Institution of Chemical Engineers Symposium Series, 1994, 136: 549-556.

[8] BATCHELOR G K. An introduction to fluid dynamics[M]. Cambridge, UK: Cambridge University Press, 2000.

[9] Wu Zongze. Mechanical Design and Practical Manual[M]. Beijing: Chemical Industry Press, 2001. 